\title{
I Wanna Hold Your Hand: The Progression of Social, Romantic and Sexual Events in Adolescent Relationships
}

\section{By Lucia F. \\ O'Sullivan, \\ Mariah Mantsun \\ Cheng, Kathleen \\ Mullan Harris \\ and Jeanne \\ Brooks-Gunn}

Lucia F. O'Sullivan is associate professor,

Department of

Psychology, University

of New Brunswick,

Canada. Mariah

Mantsun Cheng is

research associate,

Carolina Population

Center, and Kathleen

Mullan Harris is

Gillian T. Cell

Distinguished Professor, Department of Sociology, both at University of North Carolina at Chapel Hill. Jeanne BrooksGunn is Virginia and Leonard Marx Professor of Child Development and Education,

College of Physicians

and Surgeons, and

Teachers College,

Columbia University, New York.

CONTEXT: Despite the vast amount of existing research on adolescent sexual behavior, little is known about the trajectory of social, romantic and sexual events within an adolescent's relationship.

METHODS: A subsample of participants in Wave 2 of the National Longitudinal Study of Adolescent Health $(8,438$ respondents aged 12-21) provided data on the sequence of 15 social, romantic and sexual events that occurred in a recent romantic relationship. Proportions reporting each event and average relative rankings were assessed for all respondents, for different racial and ethnic groups, and for respondents who belonged to the same racial or ethnic group as their partner. Logistic regression was used to compare proportions; ordinary least square regression was used to analyze the mean sequential ranking of each event.

RESULTS: Social and romantic events, such as spending time with one's partner in a group and holding hands, were far more common than sexual events, such as touching one's partner without clothing, and typically preceded sexual events in the trajectory of relationship events. Romantic events were the most common across three of the four major U.S. racial and ethnic groups. Asian and Hispanic respondents tended to have low proportions reporting sexual events compared with white respondents. Black adolescents were the only group for whom talking about prevention of pregnancy and STDs preceded sexual events. Reports from male and female adolescents were similar.

CONCLUSION: Recognition of the diversity of relationship experiences may prompt the development of more effective interventions for adolescents who engage in risky sexual behavior.

Perspectives on Sexual and Reproductive Health, 2007, 39(2):100-107, doi: 10.1363/3910007

Most young people have been involved in at least one romantic relationship by middle adolescence, and the majority are in an ongoing relationship by late adolescence. $^{1,2}$ Adolescents report preoccupation with romantic feelings and relationships, and place considerable emphasis on the significance of these relationships in their lives. 3,4 Despite the role these early relationships play in the development of skills required for healthy adult relationships, ${ }^{5}$ research on the topic has been scarce.

Adolescents' sexual behavior, on the other hand, has garnered a wealth of research attention. Because of concerns about adolescent pregnancy, childbearing and STDs, the focus has been largely on poor individual and social outcomes. This truncated, problem-based perspective has provided little insight into normative sexual development, ${ }^{6}$ especially the array of relationship events that provide the context for many sexual interactions. ${ }^{7}$ The current study examines social, romantic and sexual events in the development of relationships among a diverse sample of U.S. adolescents.

\section{ADOLESCENT SEXUAL EXPERIENCE AND RELATIONSHIP DEVELOPMENT}

Children tend to socialize in single-sex groups until early or middle adolescence, at which point they begin to interact in mixed-sex groups. ${ }^{8}$ In this group context, adolescents develop confidence and skills in socializing with opposite-sex peers before early romantic and sexual interactions occur. In fact, romantic partners typically are drawn from and remain within these peer networks. ${ }^{9}$

Some researchers have examined milestone events, most often first intercourse, in relationship development among young adults. ${ }^{10,11}$ Others have documented adolescents' expectations about age-appropriate progression of sexual activities in relationships ("sexual timetables") ${ }^{12}$ and young adults' recall of the trajectory of their adolescent heterosexual experiences. ${ }^{13}$ Most analytic models have focused on the sequence of sexual events and have omitted other events central to relationship development, such as key social and romantic interactions. Surprisingly little is known about how social, sexual and romantic events are intertwined or coordinated over the course of adolescents' relationships.

What is known about how sexual, social and romantic events are coordinated as adolescents' relationships develop is typically based on samples of white youth. ${ }^{14}$ Yet, racial and ethnic groups likely vary in terms of key interpersonal events because of the strong influence sociocultural factors may have on adolescent development. Using a single conceptual template to represent all adolescents' experiences limits the extent to which we can understand variations in normal development. In 
a landmark longitudinal study by Smith and Udry, ${ }^{15}$ white adolescents generally progressed from romantic kissing to breast fondling, genital contact and finally intercourse, whereas black adolescents followed no predictable pattern of behavior. Indeed, many black adolescents reported intercourse without previous noncoital sexual experience.

However, in a more recent investigation, Feldman and colleagues ${ }^{13}$ found similar sequences of sexual behaviors among black, Asian, white and Hispanic adolescents. Racial and ethnic differences were apparent for the age at onset of sexual behaviors and the rate of progression through these activities, but not for the order of activities. Asian adolescents initiated each sexual activity at a later age than did adolescents in other groups. Black adolescents had the most rapid progression of sexual activities: They reported initiating kissing (typically an early sexual activity) a little before 13.9 years of age, on average, and first intercourse (typically a later sexual activity) approximately 1.6 years later, at 15.5 years of age. In contrast, the other racial and ethnic groups reported an average of 2.0-2.5 years between these two events.

The Feldman study is particularly valuable because it addressed the timing and order of first sexual interactions, not just whether an individual had experienced any of a range of sexual activities. However, the respondents were young adults (18-25 years old) recalling their adolescent experiences across relationships. It is still not known how sexual behavior unfolds in the context of a particular relationship or whether nonsexual interactions, such as romantic and social activities or events, are part of a developmental sequence central to the foundation of adolescents' relationships.

Variation in sexual experience on the basis of race and ethnicity has been documented in considerable detail. ${ }^{13,16-18}$ However, the extent to which relationship development varies on the basis of race and ethnicity is still unclear. Theoretically, cultural influences, such as family influences and media exposure, are likely to have a significant impact on the trajectory of romantic experiences. ${ }^{19}$ For example, although Asian Americans comprise a wide range of groups, many share, to varying degrees, a cultural heritage that views premarital sexual activity as a threat to family honor. ${ }^{20}$ The highly collectivistic and patriarchical character of many Asian cultures strongly discourages open sexual discourse. ${ }^{20}$ Hispanic cultures tend to emphasize family unity and allegiance, ${ }^{21}$ they also emphasize respect for authority and elders, the need for parental approval of romantic partnerships and strong endorsement of traditional gender roles. ${ }^{22}$ Black families often report discussing sexual matters in greater detail and more openly than white and Hispanic families, ${ }^{23}$ and black adolescents have been found to reject the more idealized constructs of love and romance that are commonly endorsed by white adolescents. ${ }^{19}$ In the past, researchers have often obscured racial and ethnic distinctions-for example, by classifying Hispanic indivi- duals with one racial group or another. ${ }^{24}$ Racial and ethnic differences reflect complex issues associated with cultural heritage, social inequalities and access to relevant resources. Research is needed that is more sensitive to such differences.

\section{Current Study}

Our study examines the progression of social, romantic and sexual events in the development of adolescents' relationships. Its conceptual framework links relationship development to sexual development, as well as to the broader contexts of adolescents' lives-their social interactions and sociocultural backgrounds. Our goal is to add to the literature by focusing on adolescents' reports about a recent relationship, rather than relying on young adults' retrospective reports about their experiences across relationships. We compared members of the four largest racial and ethnic groups in the United States-white, black, Asian and Hispanic-as well as adolescents of mixed race or ethnicity, a viable and growing distinction. (We refer to Hispanic and Asian ethnicities as singular identities because of the limitations of the data, but recognize the multiplicity of cultures represented by these categories.) On the basis of the literature and our conceptual framework, we explored two hypotheses. We expected that romantic and social events would precede sexual events, with wider prevalence of these experiences across all racial and ethnic groups. And we expected that Asian and Hispanic adolescents would report less sexual experience than white and black adolescents, reflecting more conservative cultural traditions, and that intercourse would occur last in their trajectories of relationship development.

\section{METHODS \\ Sample}

We used data from the National Longitudinal Study of Adolescent Health (Add Health), a longitudinal study of U.S. adolescents who were in grades 7-12 in 1995. Add Health's overall goal is to assess a complex array of health issues from adolescence through young adulthood. The sampling frame included all U.S. high schools that had an 11 th grade and at least 30 enrolled students. ${ }^{25}$ A random sample of 80 high schools was selected using stratification that was based on geographic region, urbanicity, school size, school type, percentage black and grade span.

Our sample is derived from Wave 2 of data collection, conducted in 1996. We chose this wave of data because it provides one of the most comprehensive assessments of relationship development for adolescents; subsequent waves provide assessments for young adults. Respondents completed in-home structured interviews using computer-assisted personal interviews. For more sensitive questions, such as items assessing sexual behavior, they used audio-computer assisted self-interviews (A-CASI) to listen to prerecorded questions through earphones and enter their responses directly. The Wave 
2 sample comprised 14,731 students, of whom 123 were married and 1,168 had no grand sample weights.* (Fourteen students both were married and had no grand sample weight.) When the sample was restricted to those who were not married and who had valid sample weights, it comprised 13,454 respondents (6,571 males and 6,883 females).

Respondents were asked to describe the events pertaining to at most three romantic relationships that occurred within 18 months preceding the interview. Thirty-five percent of respondents reported no "special romantic relationship in the last 18 months with any other person." Of the remaining 8,706 respondents, 73\% reported one relationship, $18 \%$ reported two and $9 \%$ reported three. To minimize recall bias, relationship events that occurred within the last 18 months are the focus of analyses. Start dates for the relationships are available for some, but not all, respondents who reported more than one relationship; therefore, for consistency, we used data on the first relationship reported, although we cannot assume that all of these relationships began during the 18-month period.

Overall, 112 respondents reported romantic relationships with same-sex partners only, 55 with both male and female partners, and 8,518 with opposite-sex partners only; the remaining 21 respondents did not report their relationship partners' sex. Our analyses focused on those reporting only relationships with the opposite sex, because the events characterizing the development of gay and lesbian relationships likely are substantially different from those for heterosexual relationships. Among these 8,518 respondents, 8,505 provided valid information about their romantic relationships. We excluded 67 whose relationships were found to have ended before the 18-month period preceding the interview; thus, the final sample included relationship reports from 8,438 respondents.

\section{Measures}

-Sample characteristics. In the following analyses, gender refers to the respondent's gender; respondent's age is measured in years. Partners' race and ethnicity were defined by the respondents. Race and ethnicity for respondents were categorized in the following manner: First, respondents were grouped on the basis of whether they indicated that they were Hispanic or non-Hispanic. Non-Hispanic respondents were further categorized by whether they reported a single racial category (white, black, Asian or other) or multiple categories. As the nonHispanic other category was very small (114 respondents and 289 partners), it was excluded from the analyses

* Respondents were missing weights either because they were missing school IDs or because they were part of a subsample of genetically related participants (and therefore not independent cases). (Source: Tourangeau R and Hee-Choon S, National Longitudinal Study of Adolescent Health: Grand Sample Weight, 1999, <http://www.cpc.unc.edu/ projects/addhealth/files/weights.pdf $>$, accessed Aug. 1, 2006.) involving race and ethnicity. Thus, race and ethnicity are represented here by five dummy variables: white, black, Asian, Hispanic, and mixed race or ethnicity (i.e., the respondent checked off multiple racial categories, not including "other").

-Relationship event sequence. Respondents were asked to identify their romantic partner by initials, which the interviewer noted. To assess the types of events that occur as the adolescent relationship evolves, the interviewers gave respondents 15 cards, each of which described a common event in adolescent relationships.

The cards described social events (i.e., "you went out together in a group," "you met your partner's parents," "you and your partner spent time alone together," "you saw less of other friends so that you could spend more time with your partner"), romantic events (i.e., "you held hands," "you kissed," "you thought of [yourself and your partner] as a couple," "you told other people that [you and your partner] were a couple," "you told each other you loved each other," "you gave each other gifts"), and sexual events (i.e., "you touched each other under your clothes or without clothes on," "you touched each other's genitals [private parts]," "you had sexual intercourse," "you talked about contraception or STDs," "your partner or you got pregnant"). Respondents then received the following instructions via A-CASI: "First take out all the cards that describe things that have not happened in your romantic relationship with [initials]. Set these cards aside. Then look through the remaining cards, and put them in the order in which they first happened in your romantic relationship with [initials]. Whatever happened first should be the first card, the thing that happened second should be the second card and so on. Don't worry if two things happened at the same time. Just pick one to put first, and put the other one after it." The events were coded ordinally from earliest (1) to latest (15).

\section{Analysis}

The proportion of respondents reporting each event was calculated, and differences in reports across groups were analyzed using logistic regression analysis. The reference group was white adolescents, because they constituted the largest group in the sample; this is a standard statistical procedure for designating reference groups. ${ }^{26}$ The unit of analysis for these calculations was individuals, and all analyses were weighted and adjusted for design effects. We used a series of logistic regression analyses on reports of the occurrence of each event (e.g., met partner's parents) by respondents' race or ethnicity. We then conducted a series of ordinary least square regression analyses on the mean sequential ranking of each event by respondents' race or ethnicity.

To examine events within relationships and to control for the influence of partner race and ethnicity, we calculated the proportions of respondents with partners of the same race and ethnicity reporting these events. Because of the high rate of occurrence of some lower-level 
romantic events, we used a more inclusive variable, which specified that romantic events in a relationship included a report of having expressed love, held hands and kissed plus one of the following: The respondent and partner told others they were a couple, thought of themselves as a couple or exchanged gifts. We also examined reports of three sexual behaviors that varied in levels of intimacy: touching under or without clothes, touching genitals and sexual intercourse.

\section{RESULTS}

\section{Sample Characteristics}

The respondents' mean age was 16.2 years (range, 12-21; standard deviation, 1.6). More than half (52\%) were female. Sixty-eight percent were white, 14\% were black, $12 \%$ were Hispanic, $2 \%$ were Asian and $3 \%$ were of mixed ethnicity. Most partners (82\%) were described as having the same racial or ethnic background as the respondent. Almost half of the relationships (49\%) were ongoing at the time of the interview. Relationships had lasted, on average, 10.2 months (standard deviation, 11.9). More than half $(56 \%)$ of respondents reported having had intercourse, and 53\% reported having had intercourse before entering the target relationship.

\section{Prevalence of Relationship Events}

Male and female adolescents' reports of relationship events were similar; data were consequently collapsed across gender. Not all youth in relationships participated in relationship events that were classified as social (Table 1). Seeing less of one's friends after being in a relationship was reported by $54 \%$ of respondents, whereas being together with their partner in a group was reported by $80 \%$. By contrast, romantic events were far more common, especially holding hands, which was reported by $94 \%$ of the youth. High proportions of respondents in all racial and ethnic groups reported that they and their partner had held hands, kissed, thought of themselves as a couple and told others that they were a couple, suggesting that both private and social recognition of relationship status are important events in adolescent relationships. Sexual events were the least commonly reported experiences for these adolescents. Getting pregnant and talking about birth control or STDs were least common, as might be expected; $8 \%$ and $40 \%$ of all respondents reported these events, respectively. Sexual touching in some form was reported by more than half of the respondents (57-63\%), whereas sexual intercourse was reported by fewer than half (45\%).

- Social events by race and ethnicity. Significantly higher proportions of white respondents than of blacks, Asians or Hispanics reported meeting partners' parents (79\% vs. 67-69\%). Higher proportions of white adolescents reported being with their partner in a group compared with black, Hispanic and mixed adolescents. Lower proportions of black adolescents than of white adolescents reported being alone with their partner (67\% vs. 78\%)
TABLE 1. Percentage of U.S. adolescents who reported selected events within a recent romantic relationship, by race and ethnicity, Wave 2 of the National Longitudinal Study of Adolescent Health, 1996

Event

$\begin{array}{llllll}\text { All } & \text { White } & \text { Black } & \text { Asian } & \text { Hispanic } & \text { Mixed } \\ (\mathrm{N}= & (\mathrm{N}= & (\mathrm{N}= & (\mathrm{N}= & (\mathrm{N}= & (\mathrm{N}= \\ 7,781) & 4,267) & 1,457) & 372) & 1,267) & 286)\end{array}$

Social

Spent time with

partner in a group

Met partner's parents

Spent time alone with partner

Saw less of friends

Romantic

Held partner's hand

Kissed partner

Thought of themselves and partner as couple

Told others they and partner were a couple

Declared love for each other

Exchanged gifts

Sexual

Touched partner under/ without clothes

Touched partner's genitals

Had sexual intercourse

Talked about birth control/STDs

Got pregnant

$\begin{array}{llllll}80.0 & 84.0 & 68.0^{*} & 81.0 & 78.0^{*} & 69.0^{*} \\ 76.0 & 79.0 & 67.0^{*} & 67.0^{*} & 69.0^{*} & 71.0 \\ 75.0 & 78.0 & 67.0^{*} & 76.0 & 74.0 & 71.0 \\ 54.0 & 55.0 & 48.0^{*} & 51.0 & 58.0 & 49.0 \\ & & & & & \\ 94.0 & 95.0 & 88.0^{*} & 89.0^{*} & 93.0^{*} & 92.0 \\ 92.0 & 93.0 & 89.0^{*} & 89.0 & 94.0 & 92.0 \\ & & & & & \\ 89.0 & 91.0 & 85.0^{*} & 89.0 & 87.0^{*} & 87.0 \\ & & & & & \\ 87.0 & 89.0 & 83.0^{*} & 77.0^{*} & 84.0^{*} & 80.0^{*} \\ 75.0 & 75.0 & 75.0 & 78.0 & 78.0 & 73.0 \\ 67.0 & 68.0 & 62.0^{*} & 69.0 & 73.0^{*} & 61.0\end{array}$

*Significantly different from percentage for whites at $\mathrm{p}<$.05. Notes: Results are weighted and adjusted for design effects. The overall $\mathrm{N}$ includes respondents who reported their race and ethnicity as non-Hispanic other. The $\mathrm{N}$ for each subgroup is an average, reflecting the total number of respondents analyzed for each event divided by the number of events.

and seeing less of their friends after entering a relationship (48\% vs. 55\%).

-Romantic events by race and ethnicity. Higher proportions of white respondents than of any other group reported telling others that they and their partner were a couple ( $89 \%$ vs. 77-84\%), and higher proportions of whites than of blacks, Asians and Hispanics reported holding hands (95\% vs. 88-93\%). Lower proportions of black and Hispanic adolescents than of their white counterparts reported that they and their partner thought of themselves as a couple and exchanged gifts, and a lower proportion of black respondents reported kissing. Interestingly, the proportions reporting that the relationship partners declared their love for one another were similar across groups (73-78\%).

-Sexual events by race and ethnicity. Black respondents, white adolescents and those of mixed race and ethnicity were similar in the proportions reporting touching under or without clothes and touching genitals, whereas Asian and Hispanic youth had lower proportions reporting these events. Black adolescents had the highest proportion reporting sexual intercourse (57\%), and Asian adolescents had the lowest proportion (32\%); blacks also had the highest proportion reporting discussion about birth control or STDs (49\%). Finally, both Hispanic and black adolescents had higher proportions reporting pregnancy compared with white adolescents (10\% and $16 \%$ vs. $6 \%$ ); no differences were found among adolescents of Asian or mixed descent. 
TABLE 2. Mean sequential ranking (and relative ranking) of relationship events among U.S. adolescents, by race and ethnicity

\begin{tabular}{lllllll} 
Event & All & White & Black & Asian & Hispanic & Mixed \\
\hline Social & & & & & & \\
Spent time with partner in a group & $3.1(1)$ & $3.0(1)$ & $3.8^{*}(1)$ & $3.0(1)$ & $3.5^{*}(1)$ & $3.0^{*}(1)$ \\
Met partner's parents & $4.6(4)$ & $4.6(4)$ & $4.2^{*}(3)$ & $5.7^{*}(6)$ & $4.9^{(5)}$ & $4.5(4)$ \\
Spent time alone with partner & $4.9(5)$ & $4.9(5)$ & $5.1(5)$ & $4.5(4)$ & $4.9(5)$ & $4.5^{*}(4)$ \\
Saw less of friends & $6.4(7)$ & $6.6(7)$ & $5.7^{*}(7)$ & $5.6^{*}(5)$ & $6.1^{*}(6)$ & $6.2^{*}(7)$ \\
Romantic & & & & & & \\
Held partner's hand & $3.5(2)$ & $3.5(2)$ & $3.8^{*}(1)$ & $3.8(2)$ & $3.6(2)$ & $3.3(2)$ \\
Kissed partner & $5.1(6)$ & $5.1(6)$ & $5.4^{*}(6)$ & $5.6^{*}(5)$ & $4.9(5)$ & $4.7(5)$ \\
Thought of themselves & & & & & & \\
$\quad$ and partner as a couple & $4.5(3)$ & $4.5(3)$ & $4.3(4)$ & $4.1^{*}(3)$ & $4.5(4)$ & $4.9(6)$ \\
Told others they and partner & & & & & & \\
$\quad$ were a couple & $4.5(3)$ & $4.6(4)$ & $4.0^{*}(2)$ & $4.5(4)$ & $4.4(3)$ & $4.4(3)$ \\
Declared love for each other & $7.4(9)$ & $7.6(9)$ & $6.7^{*}(9)$ & $6.1^{*}(7)$ & $7.0^{*}(7)$ & $7.1(8)$ \\
Exchanged gifts & $7.3(8)$ & $7.5(8)$ & $6.6^{*}(8)$ & $6.4^{*}(8)$ & $7.0^{*}(7)$ & $7.2(9)$ \\
Sexual & & & & & & \\
Touched partner under/ & & & & & & \\
$\quad$ without clothes & $8.9(10)$ & $9.0(10)$ & $8.7^{*}(11)$ & $8.9(9)$ & $8.9(8)$ & $8.4(10)$ \\
Touched partner's genitals & $10.1(12)$ & $10.2(12)$ & $9.6^{*}(12)$ & $10.3(11)$ & $10.0(10)$ & $9.5^{*}(12)$ \\
Had sexual intercourse & $11.0(13)$ & $11.3(13)$ & $10.1^{*}(13)$ & $11.0(12)$ & $10.7^{*}(11)$ & $10.2(13)$ \\
Talked about birth control/STDs & $9.4(11)$ & $9.8(11)$ & $7.9^{*}(10)$ & $9.1(10)$ & $9.3^{*}(9)$ & $8.9(11)$ \\
Got pregnant & $12.7(14)$ & $13.2(14)$ & $12.0^{*}(14)$ & $13.9(13)$ & $12.2^{*}(12)$ & $11.2^{*}(14)$ \\
\hline
\end{tabular}

*Significantly different from mean for whites at $p<.05$. Note: Lower ranking indicates earlier occurrence in relationship relative to other events.

\section{Patterns of Relationship Development}

- Overall sample. Relative rankings provide information regarding the typical order in which events occurred during the development of the relationship for all respondents and across groups (Table 2). Generally, the more common the reports of an event, the earlier it occurred in the trajectory of relationship development. As hypothesized, romantic and social events occurred before sexual events. Being together with one's partner in a group and holding hands received the lowest average ranking, indicating that these events occurred earliest in relationships. These events were followed by private and social identification as a couple, which received equal average rankings. The next items in the sequence were meeting a partner's parents, being alone with a partner and kissing. Ranking at the end of the sequence were sexrelated items: touching under or without clothes, talking about birth control and STDs, touching genitals and intercourse. Thus, the sequence of sexual events progressed from less to more intimate forms.

\begin{tabular}{|c|c|c|c|c|}
\hline Event & $\begin{array}{l}\text { Both white } \\
(\mathrm{N}=3,771)\end{array}$ & $\begin{array}{l}\text { Both black } \\
(\mathrm{N}=1,265)\end{array}$ & $\begin{array}{l}\text { Both Asian } \\
(\mathrm{N}=221)\end{array}$ & $\begin{array}{l}\text { Both Hispanic } \\
(\mathrm{N}=881)\end{array}$ \\
\hline Romantict & 69.8 & 65.9 & 68.0 & 71.9 \\
\hline Sexual & $66.0 * *$ & $71.5 * *$ & $53.0^{* *}$ & $56.5 * *$ \\
\hline Touched partner under/without clothes & 63.5 & 63.6 & 50.8 & 50.2 \\
\hline Touched partner's genitals & 57.9 & 55.3 & 45.3 & 45.2 \\
\hline Had sexual intercourse & 41.5 & 56.8 & 31.6 & 42.7 \\
\hline
\end{tabular}

**Significantly different from proportion reporting romantic events at $\mathrm{p}<.01$. + Declared love for each other, held partner's hand and kissed partner plus one of the following: told others they and their partner were a couple, thought of themselves and their partner as a couple or exchanged gifts.
-Racial and ethnic variations. For most of the racial and ethnic groups, being with one's partner in a group was ranked as occurring first in the relationship, followed by holding hands; for blacks, these events were jointly ranked first. Either private or social identification as a couple followed. These relationship events were followed by meeting a partner's parents, being alone with a partner and kissing. There was relatively little variation between groups for these early events. Mutual expressions of love occurred earlier for black, Asian and Hispanic adolescents than for whites.

For all groups except black adolescents, the sequence of sexual events was as follows: touching under or without clothing, talking about birth control or STDs, touching genitals, sexual intercourse and pregnancy. For black respondents, this sequence differed in that talking about birth control or STDs preceded all other sexual events. Asian and Hispanic respondents reported the fewest relationship events before intercourse.

Among respondents in couples with partners of the same race and ethnicity, approximately two-thirds (66$72 \%$ ) of each group reported some type of romantic event (Table 3). For every group except blacks, a greater proportion of participants reported romantic events than reported sexual events. (The number of relationships in which both partners were of mixed descent was too small for analysis.)

\section{DISCUSSION}

In line with the conceptual framework guiding this study, we argued that the study of relationship development should incorporate social, romantic and sexual events, rather than sexual events alone. Indeed, we found that each of these types of events was well integrated into relationship development. In particular, adolescents' being together with their partner in a group, holding hands, thinking of themselves and their partner as a couple and telling others that they and their partner were a couple were common events across groups and occurred early in relationship development. Our findings suggest that adolescents' relationships incorporate some of the key relationship building blocks noted by others that help individuals gain confidence with members of the opposite sex, including increased awareness of the nuances of interpersonal exchanges and communicating and negotiating one's intimate needs and interests. ${ }^{27}$

However, the social events assessed here were by no means universal. Between $54 \%$ and $80 \%$ of the sample indicated that these events were part of their relationship development sequence. This finding suggests that social events are not necessarily or fundamentally precursors of sexual activity. Furthermore, some respondents may have considered other social events not assessed here, such as talking on the phone or sitting with their partner in class, key to establishing their relationships. Extensive formative research is required to ensure comprehensiveness in the milestone events assessed. 
Romantic events, including holding hands, kissing, and privately and socially acknowledging themselves and their partner as being a couple, were also common across groups. Our findings suggest that within a particular relationship, adolescents might first establish a secure base through romantic interactions to ensure that their partner is consistent, available and responsive before venturing to more intimate forms of sexual interactions. The relative rankings support this conclusion, as they reveal a steady progression of social and romantic intimacy before sexual interactions were initiated.

Variations in patterns of relationship development emerged for the major U.S. racial and ethnic groups. Social scientists disagree over the origin and meaning of racial and ethnic differences in sexual practices. ${ }^{16}$ Without in-depth cultural analyses, we can only speculate about how to interpret differences. Notably, lower proportions of black respondents in romantic relationships reported social and romantic events, such as being alone with their partner, exchanging gifts, kissing and thinking of themselves and their partner as a couple. Coates ${ }^{19}$ has argued that black adolescents may eschew romantic norms in favor of more pragmatic and self-defensive norms. Doing so may be part of a larger oppositional framework that facilitates resistance to the prejudice, discrimination and rejection that black people in the United States often face. Anderson ${ }^{28}$ found that urban black male adolescents derided those involved in romantic, rather than strictly sexual, relationships. Alternatively, and perhaps more likely, the event options provided in the study may reflect a dominant, white romantic narrative, while failing to capture narratives familiar within other cultures. (White adolescents were more likely than black, Hispanic, Asian and mixed groups to report that they and their partner had held hands, told others that they and their partner were a couple and met their partner's parents, for instance.) In one study of Hispanic youth, a boyfriend's acts of possessiveness and jealousy constituted public demonstrations that he was in love with his girlfriend. ${ }^{29}$ Of note, all groups were similar in rates of couples' professing love to each other. Thus, communicating love to a partner might be one common theme among all adolescents, a shared script for developing relationships.

However, this study revealed far more similarities overall than differences between the major U.S. racial and ethnic groups. For all groups, relationships typically began by adolescents' and their partners' hanging out together in a group, holding hands and establishing themselves as a couple. In fact, relatively little happened before they identified themselves as a couple, which suggests the importance of this shared understanding before intimacy evolves further. Contrary to findings from an early study, ${ }^{15}$ black adolescents differed from the others only in that they talked about birth control or STDs before any other sexual events occurred; we found that higher proportions of black adolescents than any of other group reported talking about birth control or STDs. These findings have important implications for prevention interventions-many youth appear to discuss prevention options after the need for them has arisen, as also noted elsewhere, ${ }^{30}$ increasing their chances of facing the adverse consequences associated with unprotected sexual intercourse. Promoting healthier sexual interactions among adolescents requires interrupting this sequence of events in a way that permits the introduction of contraception and disease prevention by one or both partners. A focus on open exchange and clear communication need not disrupt the development of intimacy.

Using the Add Health data set, Carver and colleagues ${ }^{1}$ found that Asian adolescents were less likely to report romantic relationships in general compared with the other major racial and ethnic groups; in our study, Asian respondents and their partners were less likely than whites to tell others that they and their partner were a couple. These findings support a broader cultural-level norm of fewer opposite-sex interactions and less social support for dating for Asian youth. ${ }^{17}$ We expected that Asian and Hispanic adolescents would report the least sexual experience, with intercourse occurring latest in their trajectories of relationship development, and we found mixed support for our hypothesis: Asian and Hispanic respondents were less likely to report sexual events, but among those who did report sexual events, the events progressed in a manner similar to that of white and black adolescents. These data do not inform us about the rate at which the relationship developed, as information regarding the timing of each event in the sequence was not available; all events could have occurred on one or across many occasions. The data do, however, inform us about the relative position of events in the trajectory that constitutes relationship development among these respondents.

\section{Limitations}

As is true of most sexuality research, the analyses were based on cross-sectional, self-reported data and, hence, are subject to the biases inherent in these methods, such as the possibility of underreporting of sexual events. However, the remarkable consistency across groups in reports of the events comprising relationship development gives us confidence in the validity of reports. In addition, the generalizability of the findings is limited to in-school youth participating in heterosexual relationships, as the Add Health sampling frame does not include school dropouts. Further, some of the measures employed were imperfect; for example, the number of event cards to sort was limited, and no provision was made for reporting of simultaneous events. We found few differences between male and female reports; future research using intact couples' data might bring more such differences to light.

Another limitation is that respondents may not have fully understood the question regarding whether they had had a recent "romantic relationship," because the term was not 
defined in the interview. However, respondents were asked a series of questions regarding these relationships, were given the card-sorting task and then were given the option to reconsider any individual named as a romantic partner. Moreover, they were asked a series of questions about nonromantic but sexual relationships (not described here). These features gave us some assurance that the respondents made consistent distinctions between types of relationships and that only relationships that respondents considered romantic were included in the analyses.

Our data were derived from in-home interviews in 1996. Although these data represent the most comprehensive assessment of relationship development among U.S. adolescents, important changes may have occurred since that time for which we cannot account. Finally, the sample size for Asian adolescents was far smaller than those for the other three main racial and ethnic groups. This may have reduced the power to detect differences between groups.

\section{Conclusion}

Bearing in mind these limitations, we believe that the current study adds to the literature in a number of ways. First, this study reflects a careful treatment of racial and ethnic differences in the progression of heterosexual relationships during adolescence. Second, a long-standing criticism of the field has been the neglect of the range of possible trajectories in relationship development in favor of studies on first intercourse and first contraceptive usage. ${ }^{31}$ Our study places a number of sexual experiences in the wider context of relationship development. Third, whereas previous analyses describe individuals' experiences across relationships, ${ }^{32}$ our study examines the progression of events within a particular recent relationship.

Our findings suggest a number of implications for program planning, as well as health and clinical interventions. Romantic relationships play powerful roles in teaching interpersonal skills related to intimacy, providing emotional support and security, and validating youths' sense of self-worth. ${ }^{33}$ Educators can facilitate the acquisition of these key emotional and interpersonal capabilities through relationship skills programs and comprehensive sexuality and relationship education. ${ }^{5}$ In addition, recognition of the diversity of relationship experiences may prompt the development of more effective interventions for adolescents who engage in risky sexual behavior. It also seems essential to understand as much as possible the sequence of social, romantic and sexual events that take place in order to identify key points of intervention. For instance, it is valuable to know that discussions about preventing pregnancy and disease generally take place after high levels of sexual intimacy have been reached. On the other hand, it is useful to know that programs designed to increase parents' monitoring of their child's time alone with a partner will likely achieve little if most early involvement occurs in group contexts.
We have taken a first step in describing the complex interplay between romantic, social and sexual events in the development of heterosexual relationships among adolescents. This study contributes to a growing body of research that characterizes adolescent relationships not simply in terms of precursors to adult relationships or arenas for developing critical interpersonal skills, but as meaningful intimate experiences in their own right. Our findings call for culturally sensitive approaches to services and interventions addressing the sexual and romantic lives of adolescents.

\section{REFERENCES}

1. Carver $\mathrm{K}$ et al., National estimates of adolescent romantic relationships, in: Florsheim P, ed., Adolescent Romantic Relations and Sexual Behavior: Theory, Research and Practical Implications, Mahwah, NJ: Lawrence Erlbaum Associates, 2003, pp. 23-56.

2. Collins WA and Laursen B, Adolescent relationships: the art of fugue, in: Hendrick C et al., eds., Close Relationships: A Sourcebook, Thousand Oaks, CA: Sage, 2000, pp. 59-69.

3. Feiring C, Gender identity and the development of romantic relationships in adolescence, in: Furman $\mathrm{W}$ et al., eds., The Development of Romantic Relationships in Adolescence, New York: Cambridge University Press, 1999, pp. 211-232.

4. O'Sullivan LF and Brooks-Gunn J, The timing of changes in girls' sexual cognitions and behaviors in early adolescence: a prospective, cohort study, Journal of Adolescent Health, 2005, 37(3):211-219.

5. Barber BL and Eccles JS, The joy of romance: healthy adolescent relationships as an educational agenda, in: Florsheim P, ed., Adolescent Romantic Relations and Sexual Behavior: Theory, Research and Practical Implications, Mahwah, NJ: Lawrence Erlbaum Associates, 2003, pp. 355-370.

6. Collins WA, More than myth: the developmental significance of romantic relationships during adolescence, Journal of Research on Adolescence, 2003, 13(1):1-24.

7. Brooks-Gunn J and Furstenberg FF, Jr., Adolescent sexual behavior, American Psychologist, 1989, 44(2):249-257.

8. Maccoby EE, The Two Sexes: Growing Up Apart, Coming Together, Cambridge, MA: Harvard University Press, 1998.

9. Furman W, Friends and lovers: the role of peer relationships in adolescent romantic relationships, in: Collins WA and Laursen B, eds., Relationships as Development Contexts: The Minnesota Symposia on Child Psychology, Relationships as Development Contexts, Vol. 30, Mahwah, NJ: Lawrence Erlbaum Associates, 1999, pp. 33-154.

10. Cate RM and Koval JE, Heterosexual relationship development: is it really a sequential process? Adolescence, 1983, 18(71):507-514.

11. Lewis RA, A developmental framework for the analysis of premarital dyadic formation, Family Process, 1972, 11(1):17-48.

12. Rosenthal DA and Smith AMA, Adolescent sexual timetables, Journal of Youth and Adolescence, 1997, 26(5):619-636.

13. Feldman SS et al., Interpersonal context as an influence on sexual timetables of youths: gender and ethnic effects, Journal of Research on Adolescence, 1999, 9(1):25-52.

14. Thigpen JW et al., Normative sexual behavior of African American children, in: Bancroft J, ed., Sexual Development in Childhood, Bloomington, IN: Indiana University Press, 2003, pp. 241-254.

15. Smith EA and Udry JR, Coital and non-coital sexual behaviors of white and black adolescents, American Journal of Public Health, 1985, 75(10):1200-1203.

16. Furstenberg FF, Jr., et al., Race differences in the timing of sexual intercourse, American Sociological Review, 1987, 52(4):511-518. 
17. Grunbaum JA et al., Prevalence of health risk behaviors among Asian American/Pacific Islander high school students, Journal of Adolescent Health, 2000, 27(5):322-330.

18. Santelli JS et al., Multiple sexual partners among U.S. adolescents and young adults, Family Planning Perspectives, 1989. 30(6):271-275.

19. Coates DL, The cultured and culturing aspects of romantic experience in adolescence, in: Furman W et al., eds., The Development of Romantic Relationships in Adolescence, New York: Cambridge University Press, 1999, pp. 330-363.

20. Okazaki S, Influences of culture on Asian Americans' sexuality, Journal of Sex Research, 2002, 39(1):34-41.

21. Flores E et al., Sociocultural beliefs related to sex among Mexican American adolescents, Hispanic Journal of Behavioral Sciences, 1998, 20(1):60-82.

22. Torres JB, Masculinity and gender roles among Puerto Rican men: machismo on the U.S. mainland, American Journal of Orthopsychiatry, 1998, 68(1):16-26

23. O'Sullivan LF et al., Mother-daughter communication about sex among urban African American and Latino families, Journal of Adolescent Research, 2001, 16(3):269-291.

24. Roye CF, Condom use by Hispanic and African-American adolescent girls who use hormonal contraception, Journal of Adolescent Health, 1998, 23(4):205-211.

25. Harris KM et al., National Longitudinal Study of Adolescent Health: Study Design, 2004, <http://www.cpc.unc.edu/projects/addhealth/ design>, accessed Aug. 1, 2006.

26. Meyers LS et al., Applied Multivariate Research: Design and Interpretation, Thousand Oaks, CA: Sage, 2005.

27. Dunphy D, The social structure of urban adolescent peer groups, Sociometry, 1963, 26(2):230-246

28. Anderson E, Streetwise: Race, Class and Change in an Urban Community, Chicago: University of Chicago Press, 1990.
29. Dietrich LC, Chicana Adolescents: Bitches, 'Ho's, and Schoolgirls, Westport, CT: Praeger, 1998.

30. McKee MD et al., Perspectives on confidential care for adolescent girls, Annals of Family Medicine, 2006, 46(6):519-526.

31. Brooks-Gunn J and Paikoff R, Sexuality and developmental transitions during adolescence, in: Schulenberg J et al., eds., Health Risks and Developmental Transitions During Adolescence, Cambridge, UK: Cambridge University Press, 1997, pp. 190-219.

32. Montgomery MJ and Sorell GT, Love and dating experience in early and middle adolescence: grade and gender comparisons, Journal of Adolescence, 1998, 21(6):677-689.

33. Furman W and Wehner EA, Romantic views: toward a theory of adolescent romantic relationships, in: Shulman S and Collins WA, eds., Romantic Relationships in Adolescence: Developmental Perspectives, San Francisco: Jossey-Bass, 1994, pp. 21-36.

\section{Acknowledgments}

The research on which this article is based was supported by grant K01-MH01689 from the National Institute of Mental Health and grant U01 HD37558 from the National Institute of Child Health and Human Development (NICHD) Research Network on Child and Family Well-Being. The authors thank Julia Graber, Pamela Collins and Claude Mellins for their comments on an early draft of this article. This research uses data from Add Health, a program project designed by J. Richard Udry, Peter S. Bearman and Kathleen Mullan Harris, and funded by a grant P01-HD31921 from the NICHD, with cooperative funding from 17 other agencies. Special acknowledgment is due to Ronald R. Rindfuss and Barbara Entwisle for assistance in the original design. Persons interested in obtaining data files from Add Health should contact Add Health, Carolina Population Center, 123 W. Franklin Street, Chapel Hill, NC 27516-2524.

Author contact: osulliv@unb.ca 